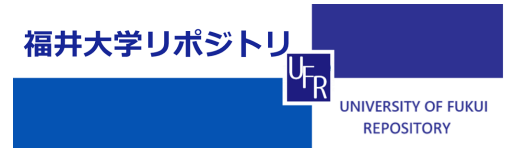

Si ze- di st ri but i on of dropl et s i n emul si ons by st at i st i cal mechani cs cal cul at i on

\begin{tabular}{|l|l|}
\hline 著者 & AOK Koi chi \\
\hline $\begin{array}{l}\text { j our nal or } \\
\text { publ i cat } \mathrm{i} \text { on } \mathrm{t} \text { i t I e }\end{array}$ & Jour nal of col I oi d and i nt er face sci ence \\
\hline vol une & 360 \\
\hline page r ange & $256-261$ \\
\hline year & $2011-10$ \\
\hline URL & ht t p: //hdl . handl e. net /10098/5194 \\
\hline
\end{tabular}




\title{
Size-distribution of droplets in emulsions by statistical mechanics calculation
}

\author{
Koichi Aoki ${ }^{*}$ \\ Department of Applied Physics, University of Fukui \\ 3-9-1 Bunkyo, Fukui, 910-8507 Japan
}

\begin{abstract}
This report is concerned with theoretical demonstration of the spontaneous emulsification which has been observed in a soft contact of nitrobenzene with water without surfactant (Electrochem. Commn. 11 (2009) 239). The demonstration is based on the model of spherical oil droplets with any size in equilibrium. The droplets are composed of the smallest droplets, the total number of which is given. An assembly of small droplets has larger surface energy than that of large ones because the surface energy is proportional to the surface area. The former has larger configurational entropy than the latter because the number of small droplets is bigger than that of the large ones. Since the free energy is determined by the competition between the surface energy and the entropy, it is not clear which assembly has lower free energy. This question was solved numerically here by statistical mechanics calculation of the size distributions, which contained only a parameter of the surface energy. The results of the computation at small number of droplets were used for deriving approximate equations for extremely large number of droplets. The size distribution was localized both to the smallest and the largest droplets. The diameter of the largest droplet was estimated from the dynamics in which coalescence by diffusion of droplets is disturbed by gravitational convection. The size then predicted was of the order of micrometer, being close to experimental values.
\end{abstract}

\footnotetext{
* e-mail kaoki@u-fukui.ac.jp, phone +81 77627 8665, fax: +81 776278750
} 
key words: emulsions; droplets formed by surface energy; statistical mechanics; distribution of particle size; phase separation

\section{Introduction}

Emulsions, being an assembly of oil droplets dispersed in aqueous phase or of aqueous droplets in oil phase, are formed temporarily when the two phases are mixed vigorously [1]. As they are left quiescent, they are gradually separated into the two phases depending on several conditions [2], or sometimes look to keep their state for a long time in the presence of surfactant, exemplified by milk or mayonnaise. Emulsions have been regarded as unstable, kinetic systems [3]. Instability mechanisms of emulsions have been categorized [3] to be Ostwald ripening [4-10] caused by combination of diffusion and formation of droplets, sedimentation or floatation called creaming [11-16] which is caused by difference between the density of droplets and that of continuous phase, aggregation or flocculation [17-21], and coalescence [22-32]. Measurements of time-varying emulsions can be categorized into a change in drop size distribution and a change in location of droplets by transport [7]. The former involves Ostwald ripening and coalescence, while the latter does creaming and aggregation. Physicochemical concern has paid to the former, especially Ostwald ripening, because it represents properties more specific to materials themselves rather than laboratory variables such as cell size and hydrodynamic conditions. Although coalescence is often considered as the most important destabilization mechanism, it depends strongly on a choice of stabilizers [10].

Emulsions are generated spontaneously when surface tension at oil|water interfaces becomes small by addition of surfactants [33-36]. Even without adding surfactants, spontaneous emulsification has sometimes been found near the interface at quiescent 
contact of oil with water [33-35,37]. The self-emulsification implies that emulsions should be more stable than the two-phase separation, and hence the emulsions would be better regarded as a quasi-equilibrium system than a dynamic one.

Implicitly time-independent emulsification has been interpreted theoretically by Lifshitz and Slyozov [38] and independently by Wagner [39]. The theory predicts the distribution of spherical droplets in radius $r$, which is normalized with the critical radius, $r_{\mathrm{c}}$, so that $p=r / r_{\mathrm{c}}$ :

$$
f(p)=\frac{81 p^{2} \mathrm{e}^{1+1 /(2 p / 3-1)}}{32^{1 / 3}(p+3)^{7 / 3}(1.5-p)^{11 / 3}} \text { for } p<1.5
$$

This has been derived on the assumption that the free energy of pressure in droplets enhanced by the curvature is compensated with an increase in concentration of the species in the continuous phase. This assumption includes ambiguity in that the enhanced free energy can also be compensated with change in surface energy, in an amount of adsorbed surfactants, or impurities such as ions. The other question is that the distribution in Eq. (1) is independent of surface energy at oil|water interfaces. Stability of emulsions has been discussed thermodynamically in the context of the interfacial energy, the ratio of concentrations of surfactant [40] and of variation of interfacial tensions with curvature of droplets [41]. Therefore, the assumptions used for the derivation of Eq. (1) cannot be realized for actual emulsions.

When interfacial tension is small, two phases are mixed well to make droplets of emulsions small. It is said that spontaneous emulsification would need negative interfacial tension [36]. This is not always true because the free energy is decreased not only by the interfacial tension but also by dispersion entropy $[42,43]$. The entropic contribution is enhanced with an increase in the number of droplets. The smaller are the droplets at a given value of the total volume of the dispersed compound, the larger is the entropy. As a simple example, we consider three smallest droplets, denoted by $\mathbf{1}$, in a continuous phase, which can coalesce to duplicate droplet, 2 , and triplet one, 3. Possible 
combinations are $(\mathbf{1}, \mathbf{1}, \mathbf{1}),(\mathbf{1}, \mathbf{2})$ and $(\mathbf{3})$. Since the sum of the interfacial area of the three droplets of $(\mathbf{1}, \mathbf{1}, \mathbf{1}), 3 \times 4 \pi r^{2}$, is larger than the area of $(\mathbf{3}), 4 \pi\left(3^{1 / 3} r^{2}\right)$, the surface energy of the former is larger than that of the latter by $3 / 3^{2 / 3}$. Therefore, the former is less stable than the latter in the view of inner energy. On the other hand, configurational entropy of the former is larger than that of the latter. A question is which has the lowest free energy of the three. In order to answer this question, it is necessary to compare each free energy of all the possible combinations. No discussion on configurational entropy has yet been performed for coexistence of various sized droplets, to my knowledge. Mixing entropy has been taken into account on the assumptions of the ideal mixing and of common size of droplets [42].

In this report, all the combinations of droplets are listed, from which their probabilities of taking the states are evaluated as a function of the surface tension and the number of the smallest droplets. The probabilities correspond to size distribution of droplets in equilibrium. Approximate expressions for the size distribution are derived at practical values of the surface tension and extremely large values of the number droplets. The possible largest size is estimated kinetically on competition of diffusion with convection.

\section{Model}

The present model is composed of oil droplets in aqueous phase. The droplets aggregate to large droplets or are decomposed into small ones to reach equilibrium among droplets. It is assumed that all the droplets are composed of $N$ smallest elementary droplets, $r_{1}$ in radius. The elementary droplet would be a hydrated oil molecule. We consider a technique of forming $K(\leq N)$ large droplets from $N$ elementary droplets. The technique is to analogize dividing $N$ shelved books into $K$ groups with $K-1$ tags. Each of $K$ groups contains $k_{n}$ books such that the summation of $k_{n}$

is $K$ and that the summation of $n k_{n}$ is $N$. There are $2^{N-1}$ ways of the arrangements, each 
of which is numbered $m$. Examples for $N=4$ are shown in Table $1 . K$ and $k_{n}$ vary with arrangement, $m$, and hence they are expressed well as $K_{m}$ and $k_{n, m}$. Then $K_{m}$ and $N$ are given by

$$
\begin{gathered}
K_{m}=\sum_{n=1}^{N} k_{n, m} \\
N=\sum_{n=1}^{N} n k_{n, m}
\end{gathered}
$$

\begin{tabular}{|l|l|l|l|l|c|l|}
\hline$K_{m}$ & $k_{1, \mathrm{~m}}$ & $k_{2, \mathrm{~m}}$ & $k_{3, \mathrm{~m}}$ & $k_{4, \mathrm{~m}}$ & arrangement & $m$ \\
\hline 4 & 4 & 0 & 0 & 0 & 6 & 1 \\
\hline 3 & 2 & 1 & 0 & 0 & $\mathbf{q}$ & 2 \\
\hline 3 & 2 & 1 & 0 & 0 & $\mathbf{q}$ & 3 \\
\hline 3 & 2 & 1 & 0 & 0 & 4 \\
\hline 2 & 1 & 0 & 1 & 0 & $\square$ & 5 \\
\hline 2 & 0 & 2 & 0 & 0 & $\mathbf{a}$ & 6 \\
\hline 2 & 1 & 0 & 1 & 0 & $\square$ & 7 \\
\hline 1 & 0 & 0 & 0 & 1 & $\square$ & 8 \\
\hline
\end{tabular}

Table 1. Arrangements of four books $(N=4)$ with $K_{m}-1$ bookmarks.

Letting the radius of a droplet made of $n$ elementary droplets be $r_{\mathrm{n}}$, the equivalence of the volumes leads to $r_{\mathrm{n}}{ }^{3}=n r_{1}{ }^{3}$. By use of the surface energy density or the surface tension, $\gamma$, at the oil|water interface, the surface energy of the droplet $r_{\mathrm{n}}$ in radius is given by

$$
\Gamma_{n}=4 \pi r_{n}^{2} \gamma=4 \pi r_{1}^{2} n^{2 / 3} \gamma
$$

By letting the chemical potential for the elementary droplet be $\mu$, the chemical potential of a droplet with radius $r_{n}$ is given by 
$u_{n}=n \mu+\Gamma_{n}=n \mu+4 \pi r_{1}^{2} n^{2 / 3} \gamma$

The inner energy of the $m$-th group in all the arrangements is given by

$U_{m}=\sum_{n=1}^{N} k_{n, m} u_{n}=\sum_{n=1}^{N} k_{n, m}\left(n \mu+4 \pi r_{1}^{2} n^{2 / 3} \gamma\right)=N \mu+4 \pi r_{1}^{2} \gamma \sum_{n=1}^{N} k_{n, m} n^{2 / 3}$

The probability of forming the $m$-th group is given by the Boltzmann distribution:

$$
\begin{aligned}
& p_{m}=A_{m} \exp \left(-\beta N \mu-4 \pi \beta r_{1}^{2} \gamma \sum_{n=1}^{N} k_{n, m} n^{2 / 3}\right) \\
& A_{m}=1 / \prod_{n=1}^{N} k_{n, m} !
\end{aligned}
$$

where $\beta=1 / k_{\mathrm{B}} T$ ( $k_{\mathrm{B}}$ : Boltzmann's constant), and the product of $k_{n, m}$ ! means the duplication of $k_{n, m}$ groups. Each of $2^{N-1}$ groups can be independently formed. The total probability, equivalent to the partition function, is given by

$$
Z=\sum_{m=1}^{2^{N-1}} p_{m}=\sum_{m=1}^{2^{N-1}} A_{m} \exp \left(-\beta N \mu-4 \pi \beta r_{1}^{2} \gamma \sum_{n=1}^{N} k_{n, m} n^{2 / 3}\right)
$$

Our aim is to obtain size distributions of droplets. The probability of finding $n$-droplets in the $m$-th group is given by

$$
A_{m} \exp \left(-\beta N \mu-4 \pi \beta r_{1}^{2} \gamma k_{n, m} n^{2 / 3}\right) / Z
$$

$n$-Droplets are included in each $m$-th group by the number of $k_{n, m}$. Thus the expected value of the number of the $n$-droplets for all the groups is expressed by 


$$
q_{n}=\frac{\sum_{m=1}^{2^{N-1}} k_{n, m} A_{m} \exp \left(-4 \pi \beta r_{1}^{2} \gamma k_{n, m} n^{2 / 3}\right)}{\sum_{m=1}^{N-1} A_{m} \exp \left(-4 \pi \beta r_{1}^{2} \gamma \sum_{n=1}^{N} k_{n, m} n^{2 / 3}\right)}
$$

This is the average number of $n$-droplet, normalized by $N$. The dimensionless averaged volume occupied by $n$-droplets is expressed by

$v_{n}=n q_{n} v_{1}=n q_{n}(4 \pi / 3) r_{1}^{3}$

\section{Computation}

The size distribution, i.e., $q_{n}$ vs. $n / N$ in Eq. (9), is a function of not only $N$ and the reduced variable $\left(\beta r_{1}^{2} \gamma\right)$ but also the auxiliary variables, $k_{n, m}$. Values of $k_{n, m}$ vary so complicated with $n, m$ and $N$ through Eq. (3) that their analytical expression cannot be derived. We resorted to numerical techniques. We enumerated the repeated permutations by inserting $K_{\mathrm{m}}$ tags into $N-1$ books in a computer memory, and counted the number of books $\left(k_{n, m}\right)$ between closest tags for a given $m$. The maximum of $N$ was 17 because of limitations of the CPU time of a personal computer. We selected $r_{1}^{2} \gamma=7.2 \times 10^{-22} \mathrm{~J}$, which corresponds to the difference in the surface tensions between water and nitrobenzene, $\gamma=0.03 \mathrm{~N} \mathrm{~m}^{-1}$ and a molecule with the diameter of water, $2 r_{1}=0.31 \mathrm{~nm}$ $\left(=18 \mathrm{~cm}^{3} / 6 \times 10^{23}\right)^{1 / 3}$. Then we have $4 \pi \beta r_{1}^{2} \gamma=2.2$. This value can vary the exponential terms in Eq. (9) largely with $k_{n, m}$ and $n$ because of $\exp \left(-4 \pi \beta r_{1}^{2} \gamma\right)=0.10$. We computed $q_{n}$ for $N \leq 17$.

Figure 1 shows the dependence of the expected values of the number of $n$-droplets on $n / N$ for some values of $N$. The smallest and the largest droplets are 
preferentially formed at the cost of middle size droplets. This preference increased with an increase in $N$. For extremely large values of $N$, particle size would be localized both to the smallest $(n=1)$ and the largest $(n=N)$ ones. Figure 2 shows variations of $q_{n}$ with $n / N$ for several values of $4 \pi \beta r_{1}^{2} \gamma$ at $N=17$. For $4 \pi \beta r_{1}^{2} \gamma \leq 0$, most of droplets are small. In contrast, the population for $4 \pi \beta r_{1}^{2} \gamma>0.05$ is occupied by the largest droplets. There are very few droplets with middle size for any value of $4 \pi \beta r_{1}^{2} \gamma$. Figure 2 also shows the size-distribution (Eq. (1)) obtained by Lifshitz and Slyozov [38] and Wagner [39], where we used $p=r_{\mathrm{n}} / r_{\mathrm{N}}=(n / N)^{1 / 3}$ on the assumption of $r_{\mathrm{c}}=r_{N}$. This distribution is roughly close to the distributions at large values of $4 \pi \beta r_{1}^{2} \gamma$ by Eq. (9), although the present concept is quite different from the assumptions in Eq. (1).

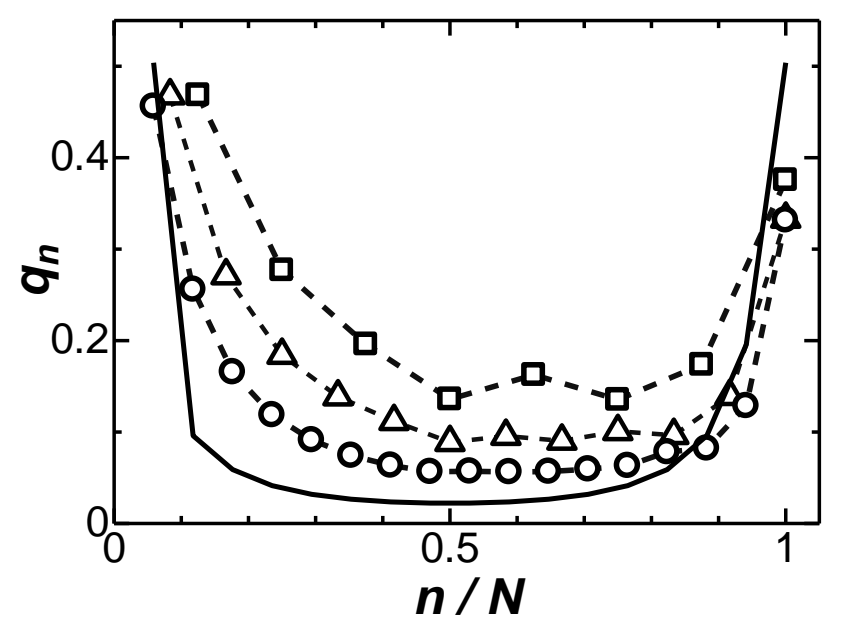

Figure 1. Variations of expected values of forming $n$-droplets with $n / N$ for $N=$ (squares) 8, (triangles) 12 and (circles) 17 at $4 \pi \beta r_{1}^{2} \gamma=2.22$, computed from Eq. (9). The solid curve was obtained from approximation, Eq. (11) and (12) for $N=17$. 


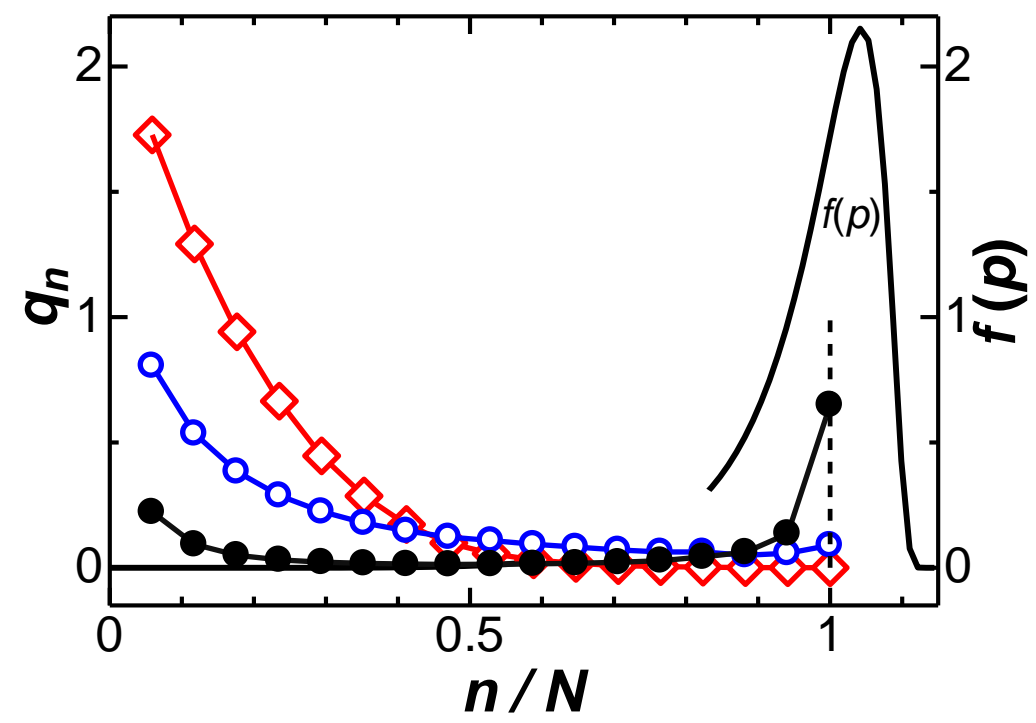

Figure 2. Variations of expected values of the number of $n$-droplets with $n / N$ for $4 \pi \beta r_{1}^{2} \gamma$ $=$ (diamonds) 0.0 , (open circles) 0.05 and (filled circles) 0.10 at $N=17$, computed from Eq. (9). The solid curve was calculated from Eq. (1) when $p=r_{\mathrm{n}} / r_{\mathrm{N}}=(n / N)^{1 / 3}$.

Figure 3 shows the distribution of volume of $n$-particles, calculated from Eq. (10) for some values of $4 \pi \beta r_{1}^{2} \gamma$ at $N=17$. The volume distribution for $4 \pi \beta r_{1}^{2} \gamma \leq 0$ is limited to small droplets, but not to the smallest one. The distribution for $4 \pi \beta r_{1}^{2} \gamma>0.05$ is mainly composed of the largest particles. Attention should be paid to the equi-volume distribution at $4 \pi \beta r_{1}^{2} \gamma=0.05$, where both the surface tension and the entropic dispersion contribute equivalently to the volume distribution. This value corresponds to $r_{1}^{2} \gamma=1.64 \times 10^{-23} \mathrm{~J}$ at $25^{\circ} \mathrm{C}$ or $\gamma=0.0007 \mathrm{~N} \mathrm{~m}^{-1}$ for molar volume of water, $2 r_{1}=0.31$ $\mathrm{nm}$. Since there is no report of typical liquid-liquid interfaces with surface tensions less than $0.0007 \mathrm{~N} \mathrm{~m}^{-1}$ [44], most emulsions should be composed of large droplets from the viewpoint of the volume fraction. Therefore removal of only largest droplets by filtering or centrifugation can decrease even small droplets efficiently. 


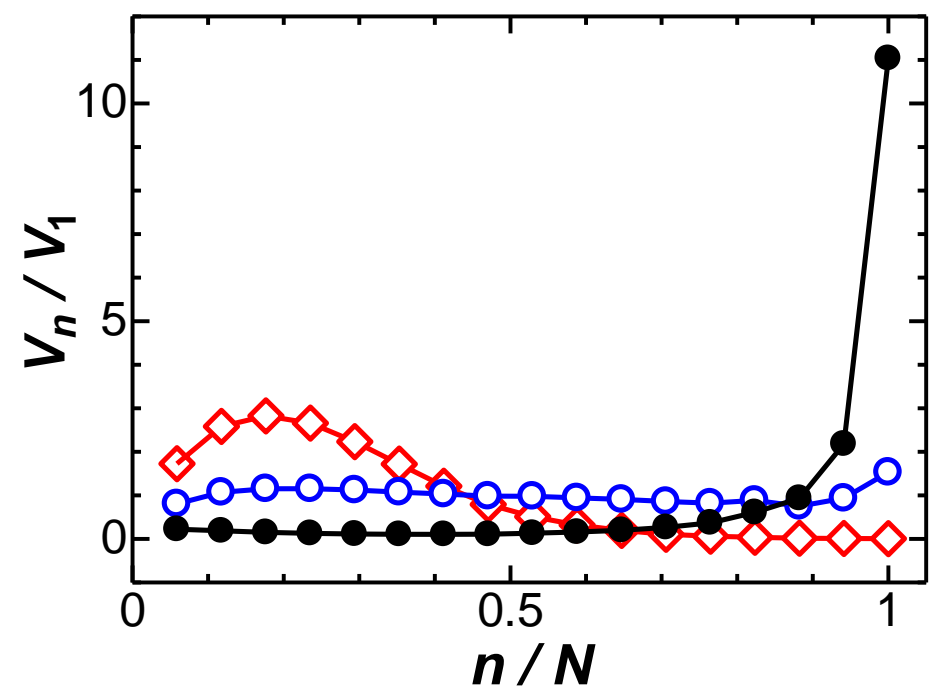

Figure 3. Variations of the expected values of the volume of $n$-droplets with $n / N$ for $4 \pi \beta r_{1}^{2} \gamma=$ (diamonds) 0.0 , (open circles) 0.05 and (filled circles) 0.10 at $N=17$.

Some combinations (not permutations) in each $m$ are identical, for example $k_{n, m}=$ $(2,1,0,0)$ for $m=2,3,4$ in Table 1 . They cannot be distinguished each other in equilibrium, and have to be removed from $Z$. It may take extremely long time to recognize identical combinations in real emulsions because all the droplets have to collide with each other. Therefore $Z$ including the identical combinations corresponds to quasi equilibrium. We tried to remove identical combinations in the computation, and compared $q_{n}$ of removing the identical combinations with that of including the combination in Fig. 4. Although the droplets in the quasi equilibrium (triangles) take the slightly smoother distribution that those in the true equilibrium (circles), the both distributions are close each other in shape. Consequently, there is no significant difference between the quasi equilibrium and true equilibrium in the view of the size distributions. 


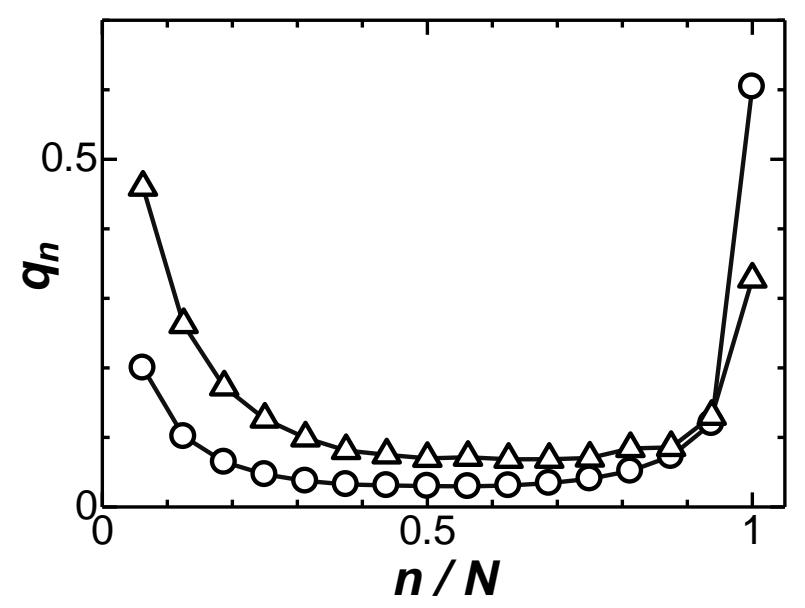

Figure 4. Variations of $q_{n}$ with $n / N$ when identical combinations were included (triangles) and removed (circles) at $4 \pi \beta r_{1}^{2} \gamma=2.22$ and $N=16$.

\section{Approximations}

Values of $N$ are of the order of the Avogadro constant in conventional emulsions, and hence the distributions for $N \leq 17$ do not represent real emulsions. Unfortunately, it was difficult for me to transform the summation of Eq. (9) into a closed analytical expression. Equation (9) for large values of $N$ was evaluated approximately by selecting the significant terms of the summations in $Z$, i.e., smaller values of the summation of $k_{n, m} n^{2 / 3}$ in Eq. (9), for example, for $K_{m}=0(n=N)$ and $K_{m}=1(n=1, N-1)$. The partition function at $K_{m}=0$ and 1 except for $\beta N \mu$ is approximated as

$$
Z^{\prime} \approx \sum_{m=1}^{N} \exp \left(-4 \pi \beta r_{1}^{2} \gamma\left\{(N-m)^{2 / 3}+m^{2 / 3}\right\}\right)
$$

Then approximations for $q_{n}$ are, depending on $n$, reduced to

$$
\begin{aligned}
& q_{1}=q_{N} \approx \exp \left(-4 \pi \beta r_{1}^{2} \gamma N^{2 / 3}\right) / Z^{\prime} \\
& q_{n} \approx 2 \exp \left(-4 \pi \beta r_{1}^{2} \gamma\left\{(N-n)^{2 / 3}+n^{2 / 3}\right\}\right) / Z^{\prime} \text { for } 2 \leq n \leq N-1
\end{aligned}
$$


The pre-exponential term "2" in Eq. (12) comes from symmetry of permutations. Equation (12) indicate that the variations of $q_{n}$ with $n$ should be symmetric with respect to $n=N / 2$ at an even number $N$. The variation of $q_{n}$ calculated from Eq. (12) for $N=17$ is shown in Fig. 1 as a solid curve. It is similar to the plot by the exact equation (circles in Fig. 1) although the valley was underestimated. Therefore the approximate equations are acceptable for values of $n$ close to 1 or $N$.

The expression for $Z^{\prime}$ is still too complicated to see the dependence of $q_{n}$ on $n / N$ clearly for an extremely large values $N$. Instead of investigating $q_{n}$, we pay attention to $q_{n} / q_{1}$. Carrying out the Taylor expansion of $(N-n)^{2 / 3}$ for $1<n<<$ yield

$$
q_{n} / q_{1}=2 \exp \left(-4 \pi \beta r_{1}^{2} \gamma\left\{(N-n)^{2 / 3}+n^{2 / 3}-N^{2 / 3}\right\}\right) \approx 2 \exp \left(-4 \pi \beta r_{1}^{2} m^{2 / 3}\right)
$$

Figure 5 shows dependence of $q_{n} / q_{1}$ on $n$ (close to unity) for several values of $4 \pi \beta r_{1}^{2} \gamma$. This equation holds even for $n$ close to $N$ because of the symmetric distribution of $q_{n} / q_{1}$ with respect to $n=N / 2$. Droplet size is localized to the smallest and the largest for large values of $4 \pi \beta r_{1}^{2} \gamma$. 


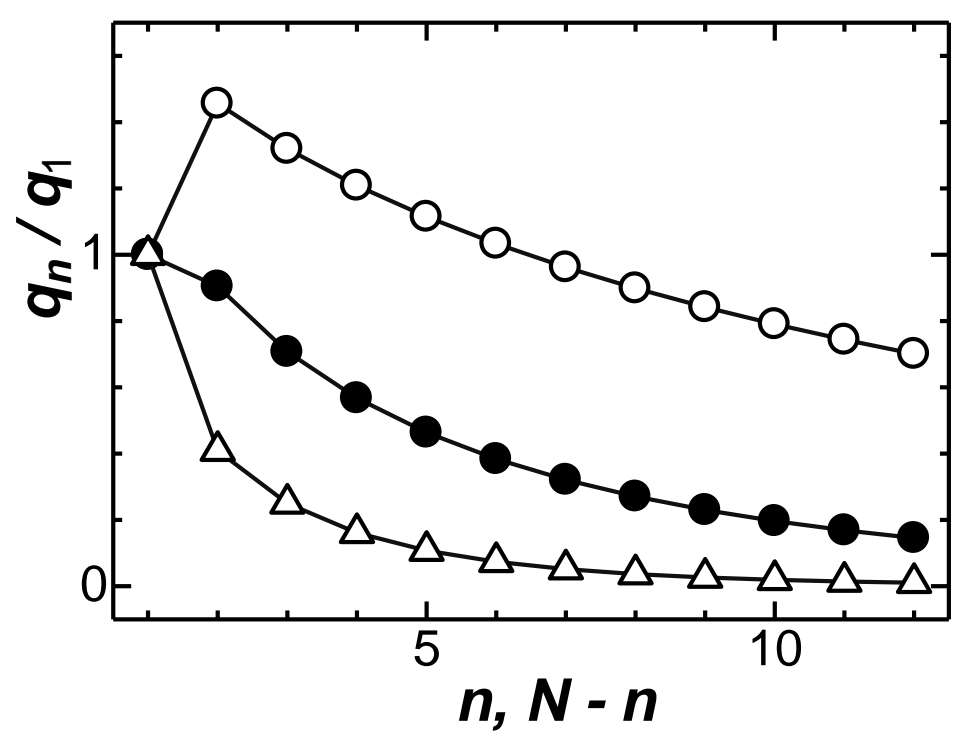

Figure 5. Dependence of $q_{n} / q_{1}$ on $n$ for extremely large values of $N$ at $4 \pi \beta r_{1}{ }^{2 / 3} \gamma=$ (open circles) 0.2 , (full circles) 0.5 and (triangles) 1, obtained from Eq. (13).

Volume concentration is more important than the number concentration in an analytical meaning. By combining Eq. (13) with Eq. (10), the volume concentration normalized by $q_{1}$ is expressed approximately by

$\begin{array}{lr}v_{n}{ }^{\prime} \equiv v_{n} / q_{1} \approx 2 v_{1} n \exp \left(-4 \pi \beta r_{1}^{2} m^{2 / 3}\right) & (\text { for } n \text { close to } 1) \\ v_{n}{ }^{\prime} \approx 2 v_{1} n \exp \left(-4 \pi \beta r_{1}^{2} \gamma(N-n)^{2 / 3}\right) & (\text { for } n \text { close to } N)\end{array}$

Values of $v_{n}{ }^{\prime}$ at $n$ close to $N$ are much larger by $N$ than those at $n$ close to 1 . Therefore the volume is mostly occupied by $N$-droplets. Since it may be slightly distributed, we obtain the domain of the preferential size distribution. The accumulated volume, which is the integration of the Eq. (14b) with respect to $n$ from $n$ to $N$ is introduced to have the form:

$$
V_{n}=\int_{n}^{N} v_{n} \mathrm{~d} n=2 v_{1} \int_{n}^{N} \xi \exp \left(-4 \pi \beta r_{1}^{2} \gamma(N-\xi)^{2 / 3}\right) \mathrm{d} \xi
$$

The integration was carried out by substitution of $(N-\xi)^{3 / 2}=x$ to yield 
$\frac{V_{n}}{2 v_{1}}=-\frac{3}{2 \alpha^{3 / 2}} \sqrt{\lambda} \mathrm{e}^{-\lambda}+\frac{3 \sqrt{\pi}}{4 \alpha^{3 / 2}} \operatorname{erf}(\sqrt{\lambda})-\frac{3}{N \alpha^{3}}\left\{1-\mathrm{e}^{-\lambda}\left(\lambda^{2} / 2+\lambda+1\right)\right\}$

where $\alpha=4 \pi \beta r_{1}^{2} \gamma$ and $\lambda=4 \pi \beta r_{1}^{2} \gamma(N-n)^{3 / 2}$. The above equation for large values of $\lambda$ and $N$ tends to $V_{N / 2}=(3 / 2) \pi^{1 / 2} v_{1} \alpha^{-3 / 2}$. The ratio becomes

$V_{n} / V_{N / 2}=-(2 / \sqrt{\pi}) \sqrt{\lambda} \mathrm{e}^{-\lambda}+\operatorname{erf}(\sqrt{\lambda})$

Figure 6 shows the variation of $V_{n} / V_{N / 2}$ with $\lambda$, which increases and reaches almost unity at $\lambda=4$. Most droplets are within the domain $4 \pi \beta r_{1}^{2} \chi(N-n)^{2 / 3}<4$, which corresponds to $n \geq N-12, n \geq N-4$ and $n \geq N-2$ for $\gamma=10,20$ and $30 \mathrm{mN} \mathrm{m}^{-1}$ at $2 r_{1}=0.31 \mathrm{~nm}$ and $25^{\circ} \mathrm{C}$.

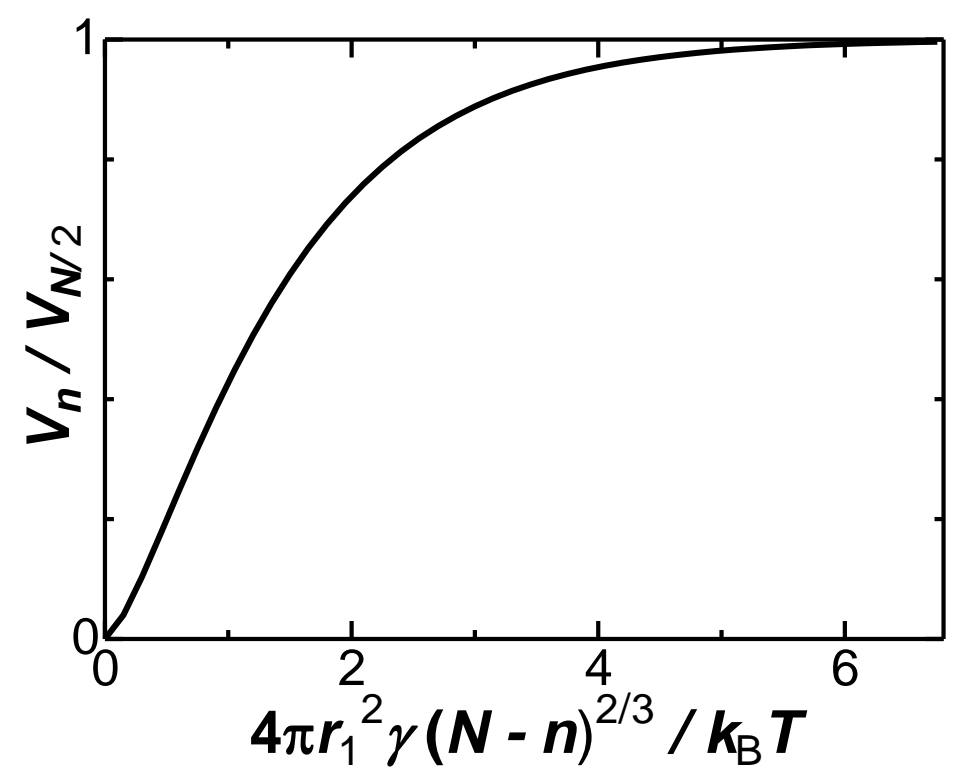

Figure 6. Variation of accumulated volume fraction with the dimensionless surface tension, $\lambda=4 \pi \beta r_{1}^{2} \gamma(N-n)^{3 / 2}$, calculated from Eq. (15). 


\section{Maximum droplets}

The largest droplet would be ideally a single oil phase into which all the droplets coalesce after a huge number of collisions. It would take extremely long time to accomplish forming a single droplet. The thermally random dispersion of droplets for a long time is really subjected to external forces such as gravity by difference in densities, motion by evaporation from surfaces of emulsions, variations of surface tensions near a container wall, stirring by mechanical vibration, gradient of temperature, and electric field. The first three factors are inevitable against attaining equilibrium. They ought to hinder the coalescence so that droplets cannot grow further to take an equilibrium size. We consider here only the effect of gravity on the disturbance of equilibrium.

The model of estimating equilibrium size is a uniform distribution of $k$ common sized droplets (radius: $r$ ) in a given volume of the emulsion, $V$. Since the volume fraction is given by $v_{\mathrm{f}}=k(4 \pi / 3) r^{3} / V$, the average distance between closest two droplets is expressed by $(V / k)^{1 / 3}=\left(4 \pi / 3 v_{\mathrm{f}}\right)^{1 / 3} r$. A condition of coalescence is to move a droplet to the closest neighboring droplets by the distance, $(V / k)^{1 / 3}-2 r$, which is reduced to

$(V / k)^{1 / 3}-2 r=\left\{\left(4 \pi / 3 v_{\mathrm{f}}\right)^{1 / 3}-2\right\} r \equiv \alpha r$

When the motion in this distance is due to diffusion for the period, $t_{\mathrm{d}}$, the thickness of the diffusion layer, $\left(D t_{\mathrm{d}}\right)^{1 / 2}$, is equal to $\alpha r$. Applying the Stokes-Einstein equation to this equality yields

$$
t_{\mathrm{d}}=\alpha^{2} r^{2} / D=6 \pi \eta \alpha^{2} r^{3} / k_{\mathrm{B}} T
$$

where $\eta$ is the viscosity of the emulsion.

The gravity causes sedimentation or buoyancy of droplets, depending on difference in densities, $\Delta \rho$ between the droplet and the continuous phase. The gravity force is balanced with Stokes' friction force when the particle moves at velocity, $v$, i.e., 
$(4 \pi / 3) r^{3} \Delta \rho g=6 \pi \eta r v$. The time of travelling $\alpha r$ at $v$ is given by

$t_{\mathrm{g}} \equiv \alpha r / v=9 \alpha \eta / 2 \Delta \rho g r$

Sedimentation prevails over equilibrium process through coalescence for $t_{\mathrm{d}}>t_{\mathrm{g}}$ to make the droplet small. On the contrary, the former is inferior to the latter for $t_{\mathrm{d}}<t_{\mathrm{g}}$ to make the droplet large. The curves of Eq.(17) and (18) are illustrated as a function of $r$ in Fig. 7. The balanced radius is at the intersection, $r_{\mathrm{m}}$, given by

$$
r_{\mathrm{m}}=\left(3 k_{\mathrm{B}} T / 4 \pi \Delta \rho g \alpha\right)^{1 / 4}
$$

This corresponds to the maximum droplet size, $r_{N}$, in the previous section. Values of $r_{\mathrm{m}}$ are 0.76 and $0.55 \mu \mathrm{m}$ for $v_{\mathrm{f}}=0.1$ and 0.01 , respectively. Experimentally controllable variables in Eq. (19) are $\Delta \rho$ and $v_{\mathrm{f}}$. Variations of $r_{\mathrm{m}}$ with $v_{\mathrm{f}}$ are shown in Fig. 8 for some values of $\Delta \rho$. Values of $r_{\mathrm{m}}$ are less than $1 \mu \mathrm{m}$ for any practical value of $\Delta \rho$ and $v_{\mathrm{f}}$.

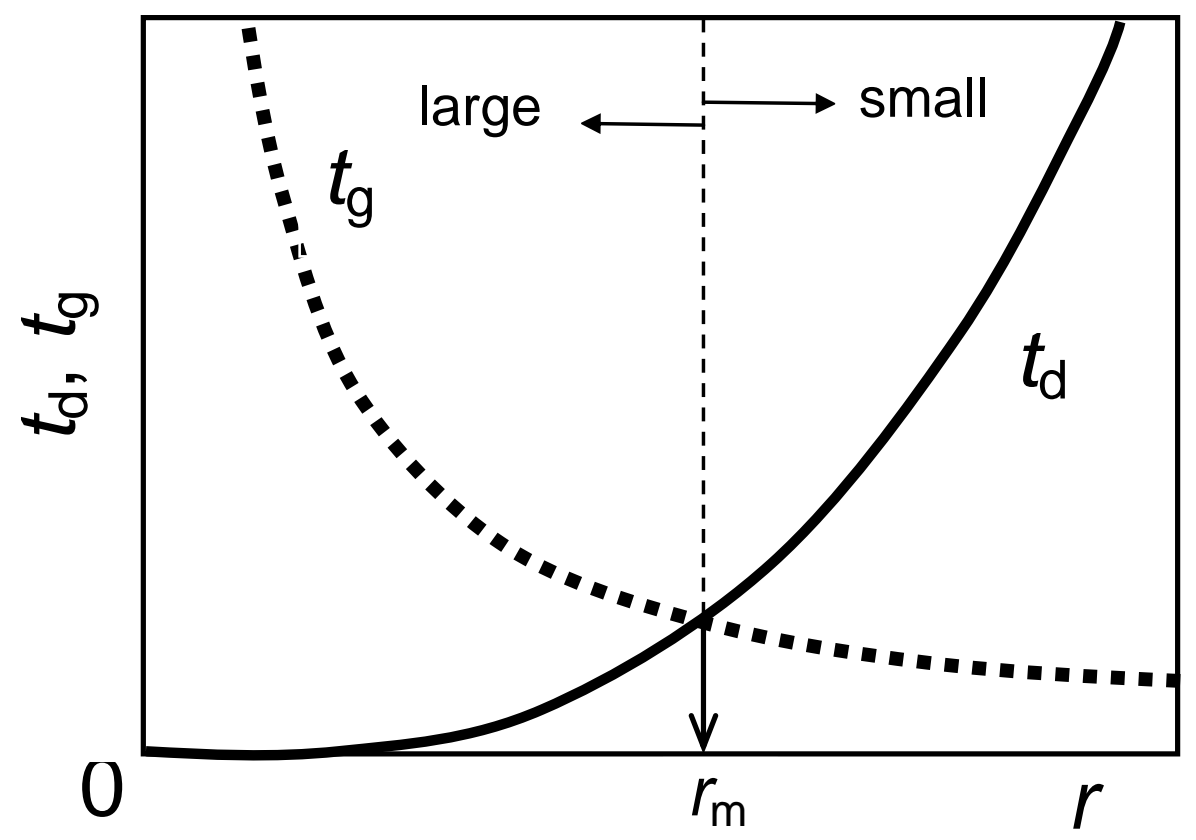

Figure 7. Predicted maximum radius, $r_{\mathrm{m}}$, from competition between diffusion and 
gravity, calculated from Eq. (17) for $t_{\mathrm{d}}$ and (18) for $t_{\mathrm{g}}$.

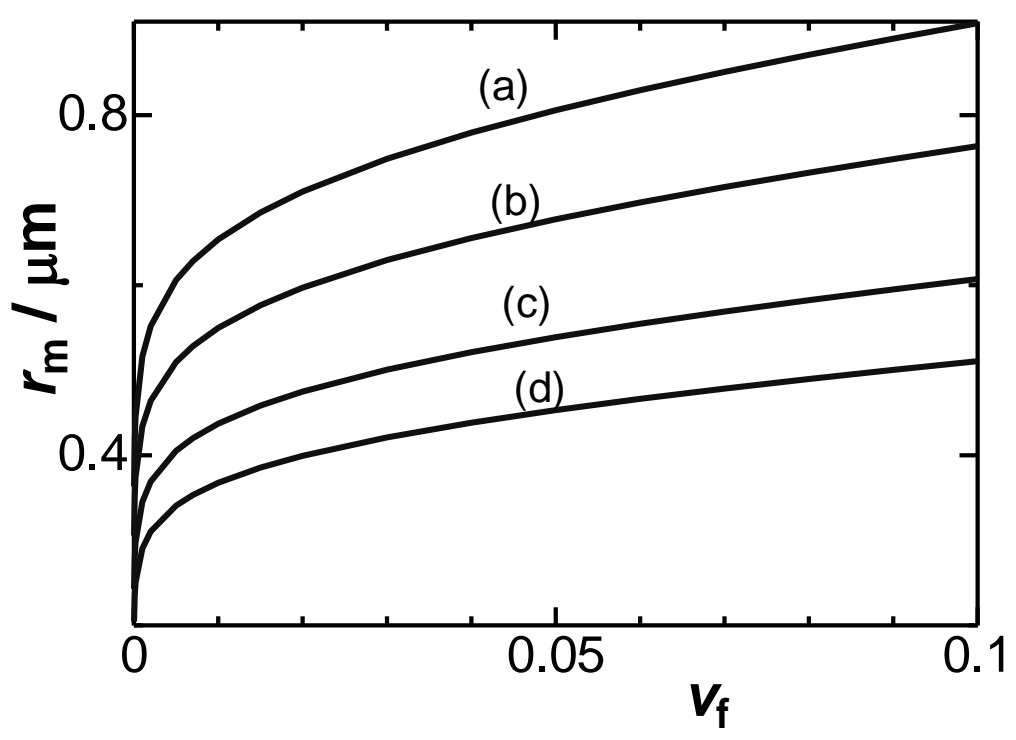

Figure 8. Variations of $r_{\mathrm{m}}$ with $v_{\mathrm{f}}$ for $\Delta \rho=$ (a) 0.1 , (b) 0.2 , (c) 0.5 and (d) $1.0 \mathrm{~g} \mathrm{~cm}^{-3}$.

The assumptions used in the above derivation are summarized here.

(i) Droplets with any size are in equilibrium by exchanging size through coalescence and decomposition.

(ii) The surface tension is independent of droplet size or mutual solvation. The independence has been extensively used for estimating the Ostwald ripening [4-10] although small curvature may vary concentrations of droplets and surfactant by the Laplace pressure.

(iii) Local fluctuation of the surface tension, as is noticed in color fluctuation on surface of soap bubbles, has been neglected.

(iv) Estimation of $t_{\mathrm{d}}$ and $t_{\mathrm{g}}$ was based on the mono-size distribution. This assumption corresponds to dependence of the quasi-equilibrium time on droplet size. 


\section{Conclusion}

The partition function of assemblies of dispersed oil droplets with any diameter was derived as a function of the surface energy on the assumption that droplets were decomposed or coalesced under equilibrium. Size distribution was evaluated numerically for small values of $N$. The results were extended to deriving approximate equations for extremely large values of $N$. The size distribution of the number of droplets was localized both to the smallest and the largest droplets at conventional values of interfacial tensions. This result is consistent with the observation of water droplets in the aqueous solution, previously reported [37]. The volume distribution was concentrated only to the largest droplets. This is of analytical importance for removing droplets.

A qualitative reason for taking a large population at the largest droplet is the minimum of the interfacial energy, whereas that at the smallest droplets is the enhancement of entropy caused by a huge number of droplets. The both contributions to the free energy are cancelled each other at droplets with middle size at conventional values of surface tension. This variation makes the volume-distribution, given by Eq. (10), be the maximum at the largest droplet.

Large droplets are subject to external forces readily enough to be far from equilibrium because their small diffusion coefficients delays exchange of droplets. When diffusion is disturbed by gravitational motion, the coalescence is hindered, and the equilibrium conditions are not satisfied for actual suspensions. The competition of diffusion with the gravitational effect yielded the droplet size of the order of micrometer. This calculation supports the observation of droplets spontaneously generated near water|nitrobenzene interfaces .

This is a revisit of theoretical estimation of size distributions of emulsions. The present concept is useful not only for emulsion polymerization and size-control of 
dispersed nanoparticles but also for electrochemically controlled ion transfer at oil|water interfaces.

\section{Acknowledgement}

This work was financially supported by Grants-in-Aid for Scientific Research (Grants 22550072) from the Ministry of Education in Japan.

\section{References}

[1] A. W. Adamson, Physical Chemistry of Surfaces, 3rd ed. John Wiley \& Sons, New York 1976, pp.491-508.

[2] E. Dickinson, Pure App. Chem. 64 (1992) 1721.

[3] E. Fredrick, P, Walstra, K, Dewettinck, Adv. Colloid Interface Sci. 153 (2010) 30.

[4] P. Walstra, Physical chemistry of foods, New York, Marcel Decker, 2003.

[5] Y. Kong, A. Nikolov, D. Wasan, Ind. Eng. Chem. Res. 49 (2010) 5299.

[6] P. Taylor, Adv. Colloid Interface Sci. 75 (1998) 107.

[7] H. W. Yarranton1, J. H. Masliyah, J. Colloid Interface Sci. 196 (1997) 157.

[8] A. S. Kabalnov, Langmuir 10 (1994) 680.

[9] M. Yu. Koroleva, E. V. Yurtov, Colloid Journal, 65 (2003) 35.

[10] M. B. J. Meinders, W. Kloek, T. van Vliet, Langmuir, 17 (2001) 3923.

[11] M. P. Aronson, Langmuir, 5 (1989) 494.

[12] T. D. Dimitrova, T. D. Gurkov, N. Vassileva, B. Campbell, R. P. Borwankar, J. Colloid Interface Sci. 230 (2000) 254.

[13] V. J. Pinfield, E. Dickinson, M. J. W. Povey, J. Colloid Interface Sci. 166 (1994) 363.

[14] S. Kumar, G. Narsimhan, D. Ramkrishna, Ind. Eng. Chem. Res. 35 (1996) 3155. [15] X. A. Wu, Energy Fuels, 22 (2008) 2346. 
[16] R. Chanamai, D. J. McClements, Colloids Surf. A, 172 (2000) 79.

[17] F. A. M. Leermakers, Y. S. Sdranis, J. Lyklema, R.D. Groot, Colloid Surf. A 85 (1994) 135.

[18] N. Diftis, V. Kiosseoglou, Food Hydrocolloids, 20 (2006) 787.

[19] M. Alexander, D. G. Dalgleish, Langmuir 21 (2005) 11380.

[20] J. Zank, P.A. Reynolds, A. J. Jackson, K.J. Baranyai, A.W. Perriman, J.G. Barker,

M.-H. Kim, J. W. White, Physica B, 385 (2006) 776.

[21] T. Sakai, K. Kamogawa, F. Harusawa, N. Momozawa, H. Sakai, M. Abe, Langmuir 17 (2001) 255.

[22] A. V. Korobko, D. van den Ende, W. G. M. Agterof, J. Mellema, J. Chem. Phys. 123 (2005) 204908.

[23] Ø. Sæther, J. Sjoblom, S. V. Verbich, N. A. Mishchuk, S. S. Dukhin, Colloids Surf. A, 142 (1998) 189.

[24] W. G. M. Agterof, G. E. J. Vaessen, G. A. A. V. Haagh, J. K. Klahn, J. J. M. Janssen, Colloids Surf. B, 31 (2003) 141.

[25] S. Arditty C.P. Whitby, B.P. Binks, V. Schmitt, F. Leal-Calderon, Eur. Phys. J. E 11 (2003) 273.

[26] M. K. Kumar, T. Mitra, P. Ghosh, Ind. Eng. Chem. Res. 45 (2006) 7135.

[27] R. Pichot, F. Spyropoulos, I.T. Norton, J. Colloid Interface Sci. 329 (2009) 284.

[28] L. Yan, K. E. Thompson, K. T. Valsaraj, J. Colloid Interface Sci. 298 (2006) 832.

[29] G. A. van Aken, F. D. Zoet, Langmuir, 16 (2000) 7131.

[30] G. A. van Aken, T. van Vliet, Langmuir, 18 (2002) 7364.

[31] S. Tcholakova, N. D. Denkov, D. Sidzhakova, I. B. Ivanov, B. Campbell, Langmuir, $21(2005) 4842$.

[32] J. Yoshida, J. Chen, K. Aoki, J. Electroanal. Chem., 553 (2003) 117.

[33] Y. A. Shchipunov, O. Schmiedel, Langmuir 12 (1996) 6443. 
[34] S. Pautot, B. J. Frisken, J.-X. Cheng, X. S. Xie, D. A. Weitz, Langmuir, 19 (2003) 10281.

[35] H. Gonza'lez-Ochoa, L. Ibarra-Bracamontes, J. L. Arauz-Lara, Langmuir 19 (2003) 7837.

[36] S. Sacanna, W. K. Kegel, A. P. Philipse, Langmuir 23 (2007) 10486.

[37] K. Aoki, M. Li, J. Chen, T. Nishiumi, Electrochem. Commn. 11 (2009) 239.

[38] I. M. Lifshitz, V. V. Slyozov, J. Phys. Chem. Solids, 19 ( 1961) 35.

[39] C. Wagner, Z. Electrochem. 65 (1961) 581.

[40] C. Wagner, Colloid Polymer Sci. 254 (1976) 400.

[41] T. Okazawa, J. Bron, J. Colloid Interface Sci. 69 (1979) 86.

[42] C. A. Miller, P. Neogi, AIChE Journal, 26 (1980) 212.

[43] E. Ruckenstein, J. C. Chi, J. Chem. Soc. Faraday Trans. II, 71 (1975) 1690.

[44] A. W. Adamson, Physical Chemistry of Surfaces, 3rd ed. John Wiley \& Sons, New York 1976, p. 39. 